\title{
Article \\ Biomechanical Analysis of Gait Compensation Strategies as a Result of Muscle Restriction
}

\author{
Jessica Beltran Ullauri ${ }^{1}$, Yasuhiro Akiyama ${ }^{1, *(\mathbb{D}}$, Shogo Okamoto ${ }^{2}$ and Yoji Yamada ${ }^{1}$ \\ 1 Department of Mechanical Systems Engineering, Mechano-Informatics and Systems, Nagoya University, \\ Nagoya 464-8603, Japan; Gabriela.Hoerig@ottobock.de (J.B.U.); yoji.yamada@mae.nagoya-u.ac.jp (Y.Y.) \\ 2 Department of Computer Sciences, Tokyo Metropolitan University, Hino, Tokyo 191-0065, Japan; \\ okamotos@tmu.ac.jp \\ * Correspondence: akiyama-yasuhiro@mech.nagoya-u.ac.jp
}

Citation: Ullauri, J.B.; Akiyama, Y.; Okamoto, S.; Yamada, Y.

Biomechanical Analysis of Gait Compensation Strategies as a Result of Muscle Restriction. Appl. Sci. 2021, 11, 8344. https://doi.org/ 10.3390/app11188344

Academic Editor: Arkady Voloshin

Received: 28 July 2021

Accepted: 2 September 2021

Published: 8 September 2021

Publisher's Note: MDPI stays neutral with regard to jurisdictional claims in published maps and institutional affiliations.

Copyright: (C) 2021 by the authors. Licensee MDPI, Basel, Switzerland. This article is an open access article distributed under the terms and conditions of the Creative Commons Attribution (CC BY) license (https:// creativecommons.org/licenses/by/ $4.0 /)$.

\begin{abstract}
The loss of muscle mass with aging and consequent muscle weakness results in compensatory gait motions. Although these compensatory motions increase the cost of walking, they appear to be an attempt by the elderly to maintain safe ambulation. However, the relationship between the affected muscles and compensatory motions in the gait cycle is unclear. This study examined gait compensation in young subjects whose muscles were weakened with Muscle Activity Restriction Taping Technique, which restricts the muscle's belly by tightening the lower limb(s). The study included different walking speeds (regular/slow) and restrictions (calf muscles/both calf and thigh muscles). It revealed that there was an active kinematic compensation chain, in which the non-restricted or less-restricted joints compensated for the affected joint to prevent foot drop, knee hyperextension in the terminal stance phase, and knee hyperflexion in the loading response phase, and to maintain the step length. Furthermore, joints could compensate for themselves when the muscles acting on the other joints were unable to assist, as observed on an ankle joint that compensated for itself to prevent foot drop when the knee and hip flexor muscles were restricted. Moreover, the observed compensation strategies agreed with a previously reported simulation on the gait compensations appearing along with muscle weakness. This study includes a comparison of these compensation strategies with those reported for the elderly. The results of this study provide an understanding of the mechanisms of gait compensation against limitations of gait ability.
\end{abstract}

Keywords: muscle restriction; joint compensation; gait analysis

\section{Introduction}

Human physical ability deteriorates with aging. An important cause is the reduction in muscle strength, mainly owing to the loss of muscle fibers and muscle crosssectional area [1-4]. In fact, a loss of at least $30 \%$ of muscle mass is expected by the age of 80 years [5,6]; this loss is greater in the lower limbs than in the upper limbs [7]. For instance, with regard to the lower limbs, significant reductions in the muscle areas of the gluteus maximus, gluteus medius, psoas major, rectus femoris (RF), vastii (VAS), biceps femoris short head (BFS), and gastrocnemius (GAS) muscles have been reported in the elderly when compared to the young [8].

With the reduction of the lower-limb muscle mass, the gait suffers from several impairments because the loss of mass causes changes in the activity magnitude of the affected muscles and their periods of action along the gait cycle. For instance, a shorter range of activity of the GAS muscle has been reported in elderly with recurrent falls [9], and a lower activity of the same muscle has been found in the elderly during the late stance phase [10]. This last might explain the elderly less-powered ankle push-off [11]. Aniansson et al. [12] reported a reduction of $25-35 \%$ in the muscle strength of the leg extensors in 70-80-year-old men. In addition, a peroneal nerve disfunction, that is a peripheral neuropathy commonly seen in the elderly, causes a lower activity or paralysis of 
the tibialis anterior (TA) muscle that in turn leads to foot drops (a gait abnormality where the forefoot drops when getting closer to the ground) [13].

Thus, the motion pattern of the lower-limb joints deviates from the normal gait pattern, partially because of the effects of muscle weakness, and partially as a result of unconscious compensation measures that the human takes to minimize the effects of muscle weakness and maintain safe ambulation [14]. For instance, an increased hip power has been reported in the elderly during the stance phase and has been suggested as a compensation for their lower-powered push-off in the late stance phase [15]. Owing to a weak TA muscle during the swing phase, the elderly exert a reduced ankle dorsal flexion; however, to maintain a safe ground clearance, the knee and/or hip joints compensate with a higher flexion [16].

According to the simulations of Van der Krogt [17] regarding the effects of muscle weakness on a lower limb, a weakened muscle can compensate for itself by exerting higher activity (as measured by electromyography), or can be compensated for by other muscles. For instance, the main leg muscle groups can compensate in the following manner: (a) The weakness of the GAS and soleus (SO) muscle groups increases the activity of the BFS muscle and decreases the activity of the TA muscle. Both the GAS and BFS muscles are responsible for knee flexion; thus, the BFS compensates for the GAS function. Additionally, the GAS and the SO muscles are responsible for plantar flexion, and the disability to exert plantar flexion is compensated by lowering the activity of the TA muscle that is responsible for the ankle dorsal flexion. (b) The weakness of the hamstring (HAM) and VAS muscles (which are responsible for knee flexion/hip extension and knee extension, respectively) increases the activity of these same muscle groups, and decreases the activity of the iliopsoas (ILPS) muscle (responsible for hip flexion). (c) The weakness of the RF muscle, which is responsible for knee extension and hip flexion, increases the activity of the ILPS and VAS muscles, and decreases the activity of the BFS muscle.

However, joint compensation motions vary widely, and the mechanisms for explaining how and when they occur have not been sufficiently investigated. Furthermore, the observation and analysis of such compensation motions are necessary to understand the mechanisms of human motion with aging.

In a previous study, we developed the Muscle Activity Restriction Taping Technique (MARTT) that restricts the transversal area of the muscle belly where it is applied and weakens the muscle activity [18]. We hypothesized that the young whose lower-limb muscles are restricted by MARTT would show compensation motions during walking such the ones seen as effect of muscle weakness that come with aging.

In this study, the compensations in the gait motion exhibited by young subjects while walking with MARTT were observed and classified, to investigate the compensation strategies. The differences in the compensation motions across the walking speeds, muscle restriction conditions, and subjects were compared and analyzed. The identified characteristics of the compensation strategies were validated based on the reported effects of muscle weakness in the gait simulations of Van der Krogt [17]. Furthermore, similarities to the compensation motions reported in the elderly were considered. Figure 1 depicts the muscles and muscles groups that are referred in this study. 


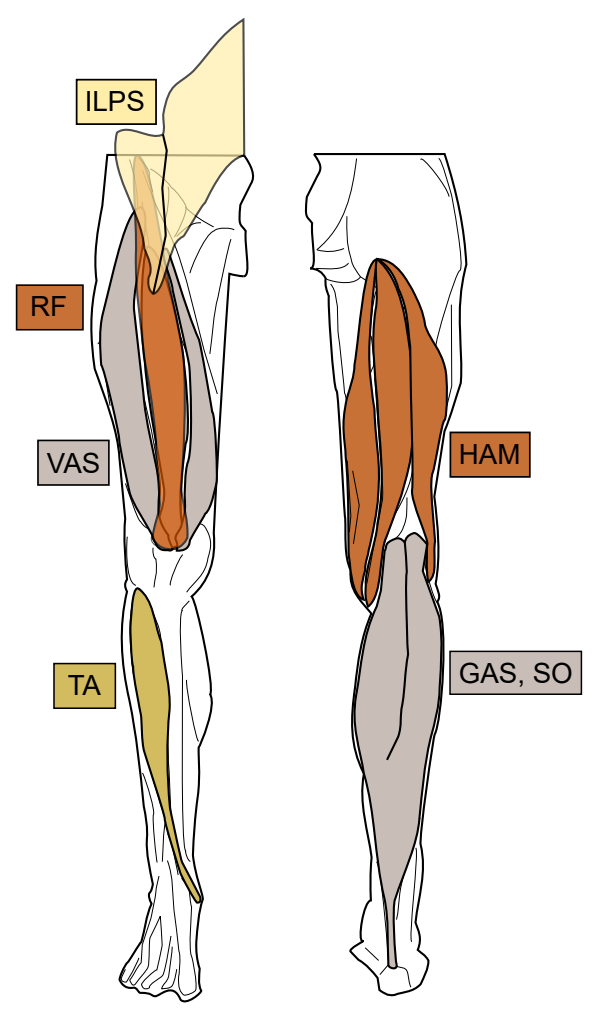

Figure 1. Location and abbreviations of muscles referred in this study. ILPS: iliopsoas muscle, RF: rectus femoris muscle, VAS: vastii muscle group, HAM: hamstring muscle group, GAS: gastrocnemius muscle, SO: soleus muscle, TA: tibialis anterior.

\section{Materials and Methods}

This study was performed with the permission of the Institutional Review Board of Nagoya University, Japan, with Approval Number 14-4. This experiment was originally performed to validate the performance of the MARTT, a wearable device that aims to modify the gait motion to that of the elderly [18].

\subsection{Subjects}

Ten healthy male young adults with a mean age of 22 years participated in this study. The mean value and standard deviation of their weights and heights were $63.9 \pm 8.9$ and $1.72 \pm 0.05 \mathrm{~m}$, respectively. Their dominant leg was the right leg.

\subsection{Apparatus}

Every gait experiment was conducted on a treadmill with a $1.4 \times 0.5 \mathrm{~m}^{2}$ walking surface (OMEGA3, Johnson Health Tech Co., Ltd., Taichung City, Taiwan). A motion capture system (MAC 3D system, Motion Analysis Corporation, Rohnert Park, CA, USA) was used to record the gait motion at a frequency of $100 \mathrm{~Hz}$. The joint patterns and subjects postures were calculated by fitting the positions of reflective markers attached to the subject to a motion module of Software for Interactive Musculoskeletal Modeling (SIMM, Musculographics Inc., Santa Rosa, CA, USA). Furthermore, four flexible force-sensing resistors (FSR-400, Interlink Electronics, Lake Forest, CA, USA) were embedded at each shoe tip and shoe sole of comfortable sport shoes, to detect heel contact $(\mathrm{HC})$ and toe off (TO) events.

The MARTT, which is a wearable device, that basically incorporates belts and force sensors, for restricting lower-limb muscles by tightening the limb [18], was used in this experiment. With MARTT, the muscle contraction was disturbed by limiting the expansion of the cross section area of the muscle. The MARTT mainly restricted the GAS and TA muscles, when it was used on the calf, and the HAM, VAS, and RF muscles, when it was used on the thigh. The same restriction force was applied to each leg, which was 
monitored with the incorporated force sensors in MARTT. The applied force corresponded to a pressure of approximately $180 \mathrm{mmHg}$, that is in the range of safe blood flow restriction (160-230 mmHg [19]) and below $200 \mathrm{mmHg}$ that could cause muscle fatigue [20].

\subsection{Protocol}

After an informed consent procedure, every subject wore a well-fitted sport suit and markers for the motion capture system. Next, the subjects were instructed to walk for a short period of time to get accustomed to the shoes and the walking path on the treadmill. The recording trials, each with a duration of $6 \mathrm{~min}$, were initiated.

In this experiment, two walking speeds (regular/slow) and two muscle restriction conditions (calf/calf and thigh) were investigated. The regular speed was set at $1.11 \mathrm{~m} / \mathrm{s}$ $(4 \mathrm{~km} / \mathrm{h})$, which is regarded as the natural walking speed of young adults [21], and the slow speed was set at $0.97 \mathrm{~m} / \mathrm{s}(3.5 \mathrm{~km} / \mathrm{h})$, which is close to the average walking speed of the elderly [22]. The restriction of the calf and thigh was applied using the MARTT. In the first condition, the MARTT was applied on the calf. It was named as the calf restriction condition (referred to as C-restriction). In the other condition, MARTT was applied on both the calf and the thigh. It was named as the calf and thigh restriction condition (referred to as CT-restriction). In total, four walking conditions were investigated, i.e., the combinations of the two levels of walking speeds and two types of restriction conditions.

In the recording session, six walking trials were recorded for each subject: the above mentioned four restricted trials, and two non-restricted trials at both walking speeds. First, the results of the non-restricted trials at the two levels of walking speeds were recorded in random order. Then, the results from the four types of restricted conditions were recorded, also in random order. Hereon, the non-restricted condition is referred to as the normal walking condition. The restriction forces applied to the muscles were monitored online to ensure that the magnitude of the restriction force applied to both legs was the same, and that it remained stable throughout the restricted trials.

\subsection{Data Processing}

The first minute of the recorded gait motion of each trial was excluded from the analysis, as it was considered as an adaptation time to the experimental conditions. Then, the position data of all markers were smoothed using a $6 \mathrm{~Hz}$ Butterworth filter. The timing of the gait events ( $\mathrm{HC}$ and $\mathrm{TO}$ ) were determined according to the ground reaction force observed at the foot soles. Every gait cycle was determined as the gait motion occurring between two consecutive HCs of the same leg.

The angles of the lower-limb joints in every gait cycle were calculated with the human motion analysis software SIMM; this software obtains the position of markers placed along the body, as recorded by the motion capture system. The joint motion patterns were normalized to a $0-100 \%$ gait cycle, and were separated into the following gait sections: loading response (first $10 \%$ ), middle stance (10-30\%), terminal stance (30-50\%), pre-swing (50-62\%), initial swing (62-75\%), middle swing (75-85\%), and terminal swing (85-100\%) [23]. Each section of the gait cycle is shown in Figure 2. Furthermore, the joint angles in every gait section were analyzed across subjects to determine the characteristics (differences) of the restricted gait (C-restriction and CT-restriction) in comparison to the normal gait. Several of the found characteristics occurred at the beginning or end of the mentioned gait sections. In that case, the first or last $5 \%$ of the corresponding section, respectively, was taken for further statistical analysis. In the section where a characteristic was identified, the respective joint angle was averaged for every subject and walking condition. The statistical significance within subjects of the characteristics in comparison to the normal gait was tested using a two-tailed paired $t$-test and compensated with the Bonferroni method.

The Institutional Review Board of Nagoya University, Japan, approved the study and registered it under approval Number, 14-4. 


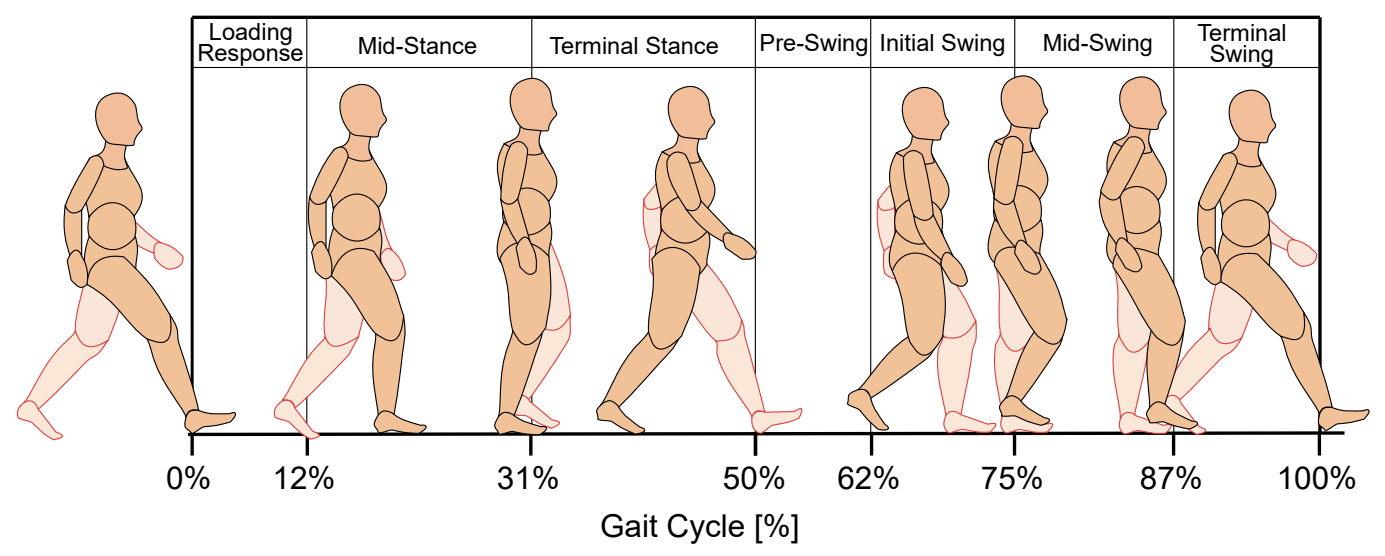

Figure 2. Human gait cycle.

\section{Results}

\subsection{Joint Compensation}

The compensation action of a joint is considered as the increment in its flexion or extension range in comparison to its normal range of motion, and works to overcome the effects of muscle weakness. The majority of the subjects ( 8 out of 10 subjects) compensated for the joint restriction with the non-restricted or less-restricted joints. This compensation behavior appeared to be independent of the order of the experimental trials and walking speeds.

In the C-restriction case, the subjects compensated for the restricted ankle motion with the non-restricted hip and/or knee joints. In the CT-restriction case, where the ankle, knee, and hip joints were restricted, the majority of subjects compensated for the ankle joint. In the remaining two subjects, one subject always compensated for the hip joint regardless of the restricted muscles, and the other exhibited no compensation and a permanent lower hip range of motion. Figure 3 shows the joint motion for the C-and CT-restrictions of the left leg of a subject; this represents the motions seen in the majority of the subjects.

In this section, the joint motion characteristics seen in every restriction type and walking speed will be described, along with the number of subjects that showed each characteristic and the significance evaluated in every subject (as specified with $p$-values).

\subsection{Joint Motion in C-Restriction}

In the ankle joint, the majority of subjects exhibited (a) higher plantar flexion at the beginning of the loading response phase, (b) higher dorsal flexion in the terminal stance phase, and (c) lower dorsal flexion during the swing phase (see these characteristics in Figure 3a). At the speed of $3.5 \mathrm{~km} / \mathrm{h}$, (a) seven $(p<0.01)$, (b) eight $(p<0.01)$, and (c) seven $(p<0.01)$ subjects presented these characteristics, respectively. Furthermore, at the speed of $4 \mathrm{~km} / \mathrm{h}$, (a) six (five with $p<0.01$ and one with $p<0.05$ ), (b) nine $(p<0.01$ ), and (c) seven (six with $p<0.01$ and one with $p<0.05$ ) subjects showed the same characteristics.

In the knee joint, all subjects showed higher knee flexion, mainly in the (a) loading response and (b) terminal stance phases, and (c) from the initial to middle swing phase (see Figure 3c). At the speed of $3.5 \mathrm{~km} / \mathrm{h}$, (a) eight (six with $p<0.01$ and two with $p<0.05)$, (b) seven $(p<0.01)$, and (c) seven $(p<0.01)$ subjects presented this characteristic, respectively. Furthermore, at the speed of $4 \mathrm{~km} / \mathrm{h}$, (a) eight $(p<0.01)$, (b) seven $(p<0.01)$, and $(c)$ seven $(p<0.01)$ subjects showed this characteristic.

In the hip joint, the subjects mainly exhibited (a) higher hip extension in the middle stance (see Figure 3e), (b) higher hip extension in the terminal stance (see Figure 3e), (c) higher hip flexion in the initial swing and (d) higher hip flexion in middle swing. At a walking speed of $3.5 \mathrm{~km} / \mathrm{h}$, (a) four $(p<0.01$ ), (b) six (five with $p<0.01$ and one with $p<0.05$ ), (c) five (four with $p<0.01$ and one with $p<0.05$ ), and (d) six $(p<0.01)$ subjects presented these characteristics, respectively. At the speed of $4 \mathrm{~km} / \mathrm{h}$, the same trend was observed in (a) ten (eight with $p<0.01$ and two with $p<0.05)$, (b) nine $(p<0.01)$, (c) seven $(p<0.01)$, and (d) eight $(p<0.01)$ subjects, respectively. 


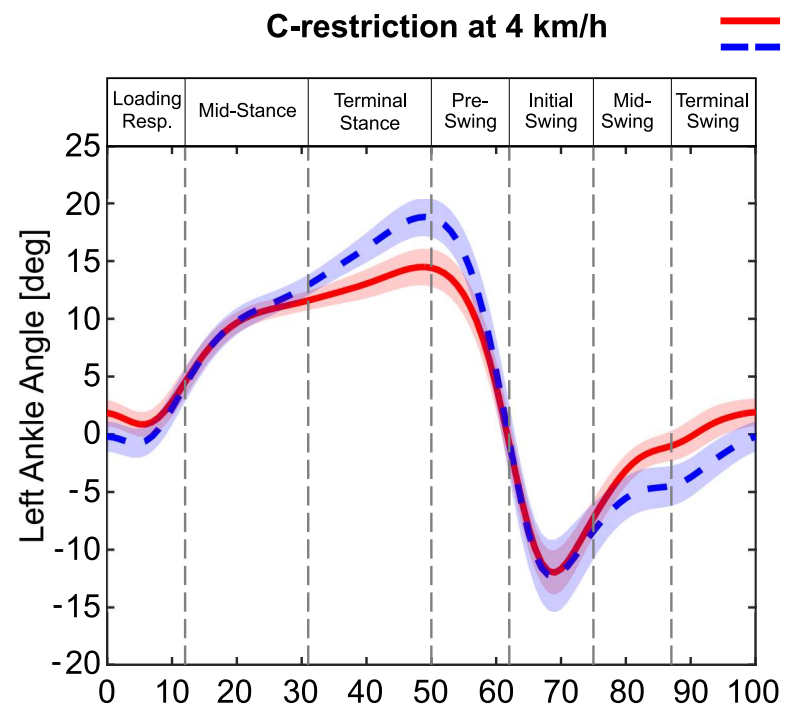

(a) Ankle motion at C-restriction: Higher plantar-flexion at loading response, higher dorsal-flexion at terminal stance and pre-swing, and higher plantar-flexion at mid- and terminal swing.

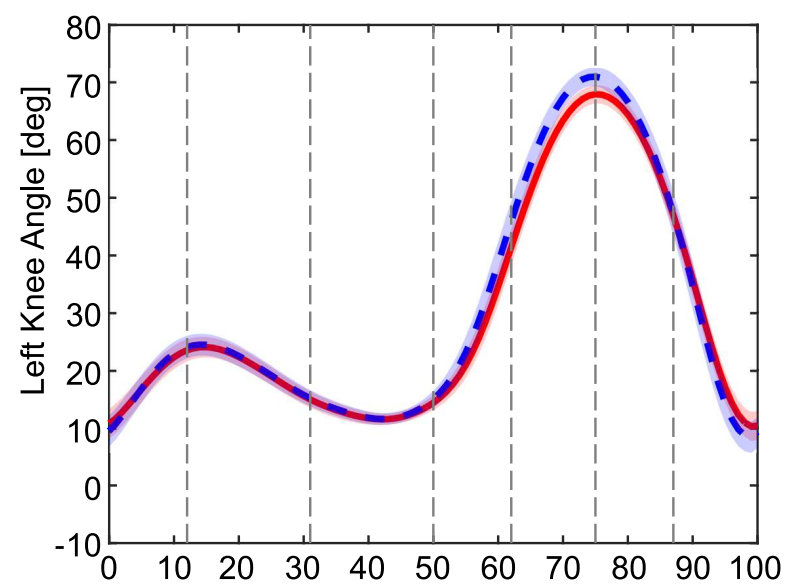

(c) Knee motion at C-restriction: Higher knee flexion at initial and middle swing.

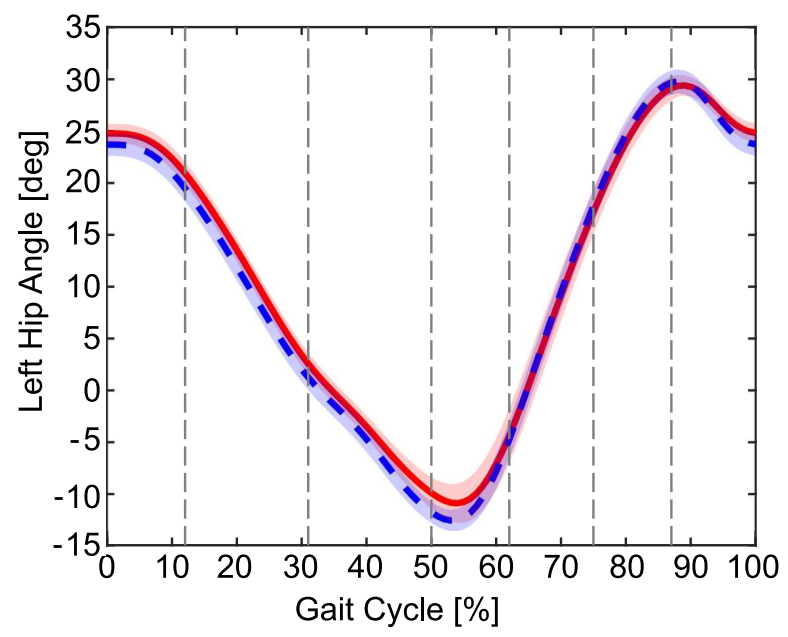

(e) Hip motion at C-restriction: Tendency to a higher hip extension from loading response until the end of terminal stance.

\section{Normal CT-restriction at $4 \mathrm{~km} / \mathrm{h}$}

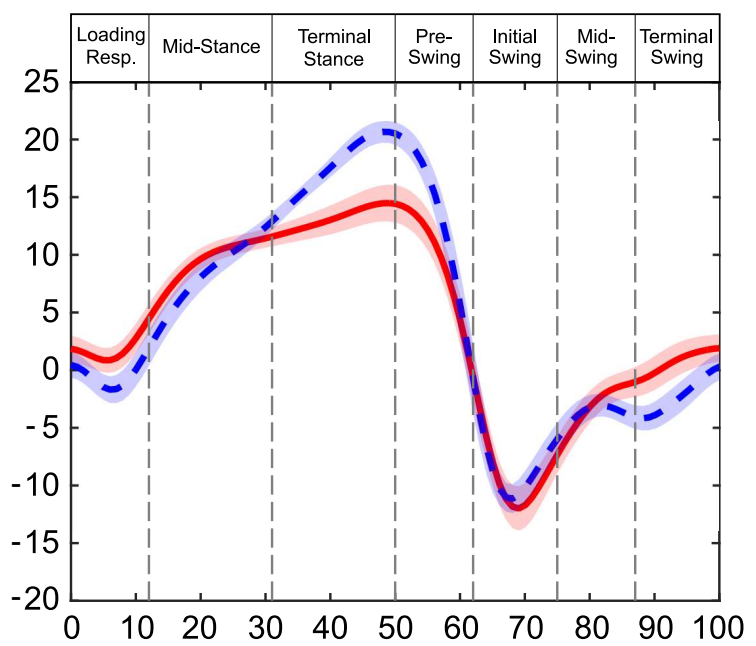

(b) Ankle motion at CT-restriction: Higher dorsal-flexion at terminal stance, pre-swing.

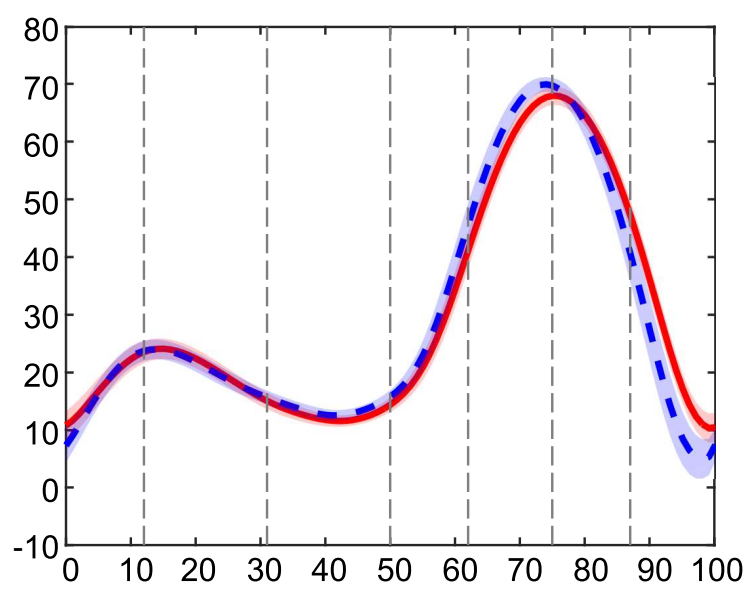

(d) Knee motion at CT-restriction: Tendency to a higher knee extension at terminal swing.

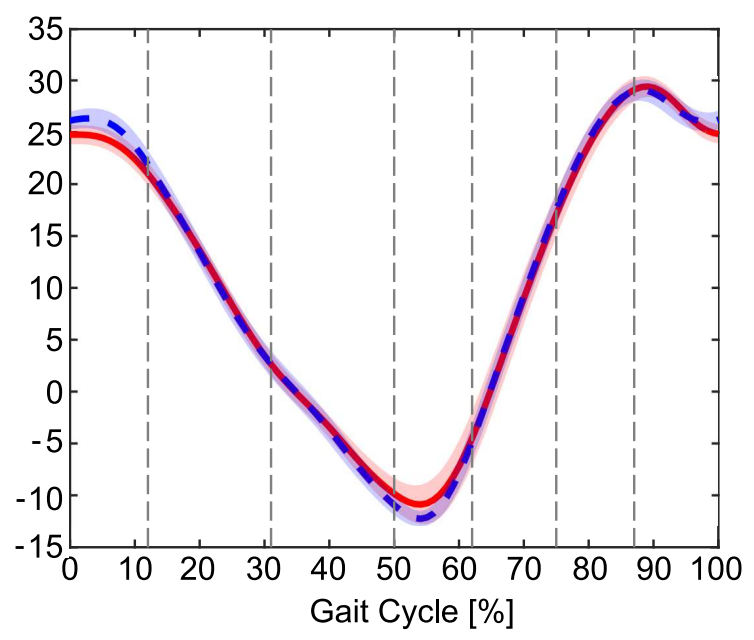

(f) Hip motion at CT-restriction: Tendency to a higher hip extension at pre-swing.

Figure 3. Joint motion of a representative subject, showing the typical motion seen in the majority of the subjects. 


\subsection{Joint Motion in CT-Restriction}

In the ankle joint, the subjects mainly presented higher dorsal flexion in the (a) terminal stance (see Figure $3 \mathrm{~b}$ ) and (b) initial and terminal swing. At the speed of $3.5 \mathrm{~km} / \mathrm{h}$, (a) eight $(p<0.01)$ and $(b)$ seven $(p<0.01)$ subjects presented higher dorsal flexion, respectively. At the speed of $4 \mathrm{~km} / \mathrm{h}$, the same trend appeared in (a) ten $(p<0.01)$ and (b) eight $(p<0.01)$ subjects, respectively. The higher dorsal flexion in the terminal stance phase was also seen in the C-restriction in the same eight subjects at the speed of $3.5 \mathrm{~km} / \mathrm{h}$ and in nine subjects at $4 \mathrm{~km} / \mathrm{h}$.

In the knee joint, seven (six with $p<0.01$ and one with $p<0.05$ ) subjects presented a higher extended knee in the terminal swing phase (see Figure $3 \mathrm{~d}$ ) at the walking speed of $3.5 \mathrm{~km} / \mathrm{h}$, and nine $(p<0.01)$ subjects at the speed of $4 \mathrm{~km} / \mathrm{h}$.

In the hip joint, the majority of the subjects exhibited (a) higher hip extension at the beginning of the pre-swing phase (see Figure $3 \mathrm{f}$ ), (b) lower hip flexion during the initial and middle swing phases, and (c) lower hip flexion in the terminal swing phase. At the walking speed of $3.5 \mathrm{~km} / \mathrm{h}$, (a) eight (seven with $p<0.01$ and one with $p<0.05$ ), (b) seven $(p<0.01)$, and (c) ten subjects (nine with $p<0.01$ and one with $p<0.05)$ presented these compensations, respectively. At the walking speed of $4 \mathrm{~km} / \mathrm{h}$, these compensation motions appeared in (a) nine $(p<0.01)$, (b) nine (eight with $p<0.01$ and one with $p<0.05$ ), and (c) seven $(p<0.01)$ subjects, respectively.

\subsection{Gait Timing}

The gait timing characteristics between subjects is shown in Table 1. As discussed in a previous article [18], the majority of subjects with the MARTT adapted their gait with a significantly lower single support phase. Several subjects also presented a significantly lower step length. However, no significant change in cadence was found.

Table 1. Gait timing between subjects. Median $\pm I Q R$ among subjects. The step length was normalized to the subject height. The information in parentheses indicates the number of subjects that experienced a significant reduction in the respective gait parameter. ${ }^{*} p<0.05$ indicates a significant reduction in comparison with normal walking.

\begin{tabular}{ccccc}
\hline Parameter & Walking Speed $[\mathbf{k m} / \mathbf{h}]$ & Normal Walking & C-Restriction & CT-Restriction \\
\hline Cadence & 3.5 & $102.71 \pm 12.34$ & $101.37 \pm 6.42$ & $100.35 \pm 8.89$ \\
[steps/min] & 4 & $107.97 \pm 7.70$ & $104.15 \pm 7.38$ & $105.46 \pm 7.79$ \\
\hline Step Length & 3.5 & $0.57 \pm 0.03$ & $0.57 \pm 0.05\left(5^{*}\right)$ & $0.57 \pm 0.04\left(4^{*}\right)$ \\
[m] & 4 & $0.60 \pm 0.03$ & $0.61 \pm 0.03\left(3^{*}\right)$ & $0.60 \pm 0.05\left(5^{*}\right)$ \\
\hline Single Support Phase & 3.5 & $64.20 \pm 5.43$ & $63.45 \pm 3.14\left(8^{*}\right)$ & $64.13 \pm 3.42\left(8^{*}\right)$ \\
[\% stride] & 4 & $66.36 \pm 4.25$ & $63.93 \pm 4.32\left(8^{*}\right)$ & $64.55 \pm 3.11\left(9^{*}\right)$ \\
\hline
\end{tabular}

\section{Discussion}

\subsection{Compensation Strategies}

As a result of the muscle restriction with the MARTT, the majority of subjects exhibited motion compensation in the non-restricted joints. This compensation behavior agrees with the results reported in the gait simulations of Van der Krogt [17], who studied the effects of muscle weakness on human gait. The simulations suggested that a weakened muscle might react by decreasing or increasing its own activity, or the activity of other muscles. In this section, we discuss the changes in the motion patterns of each joint (as mentioned in the results section) by comparing them with the compensation strategies reported in the simulations of Van der Krogt, i.e., those reported as a consequence of the weakness of certain muscles or muscle groups.

\subsubsection{C-Restriction}

In C-restriction, the mainly restricted muscles are the GAS and TA.

Effect on the ankle motion: 
According to Van der Krogt's simulations, when the TA and GAS muscles are weakened, their activity level reduces as the weakness increases. Additionally, when the GAS and SO muscle groups are weakened, the TA muscle activity decreases. The compensation motion observed in our study as shown in Figure 3a agrees with the decay in the TA muscle activity during the loading response and swing phases, where, in a normal gait, the TA muscle is known to have its most prominent activity in generating a dorsal-flexion moment [23]. The higher plantar flexion during the loading response phase observed in our experiment suggests that the movement of the foot toward plantar flexion after HC is not decelerated by a weak TA muscle, as it occurs in the normal gait. Thus, the ankle reaches a higher plantar flexion than normal before a sufficient dorsal-flexion moment is created at the ankle that moves the tibia forward over the articular surface of the talus (known as ankle rollover). During the swing phase, we observed that the ankle exhibited a lower dorsal flexion, resulting in a lower foot clearance.

The weakness of the GAS muscle was noticeable in the majority of subjects from the mid-stance to terminal stance, when the GAS in the normal gait is most active in controlling the dorsal flexion of the ankle before TO [23]. At the end of the rollover, we observed that the ankle reached a higher dorsal-flexion angle before initiating plantar flexion to prepare for TO. This agrees with the concept of a weakened GAS muscle that could not decelerate the dorsal flexion of the ankle as normal.

\section{Effect on the knee motion:}

An increased knee flexion from the loading response to mid-stance was observed in the majority of subjects (see Figure 3c), that suggests a weakness in the VAS muscles. In the normal gait, these muscles prevent knee hyperflexion in the loading response phase, and extend the knee until the middle of mid-stance [23]. Moreover, the observed higher knee flexion in the terminal stance and initial swing suggests compensation from the BFS muscle as reported in the simulations of Van der Krogt, i.e., as a compensation for the weak GAS and SO muscles. The higher activity of the BFS muscle in a terminal stance could help prevent knee hyperextension, and the higher activity in the initial swing could compensate for the lower dorsal flexion of the ankle and ensure sufficient foot clearance.

\section{Effect on the hip motion:}

Van der Krogt's simulations reported increased activity in the HAM muscles as compensation for the weakness of the GAS muscle. In agreement with this compensation, we observed a higher hip extension from the loading response to the middle of the midstance (see Figure 3e), where in normal gait the semimembranosus and semitendinosus muscles (HAM muscles) are active to extend the hip. In addition, we observed an increased hip extension at the end of the terminal stance phase, which suggests a weakness in the tensor fascia lata that limits the hip extension before the hip starts to flex in the pre-swing in the normal gait. Moreover, the increased hip flexion observed in the initial swing suggests increased activity in the ILPS muscle, also seen in Van der Krogt's simulations, as a compensation for the GAS muscle weakness. This could be an action to compensate for the lack of the hip flexion moment that is normally generated in pre-swing and is usually enough to drive the hip passively during the initial swing phase [23]. As a result, the extra hip flexion would support the above-mentioned compensation of the knee, and contribute to the foot clearance.

Summary of C-restriction: When the muscles at the calf area were restricted (Crestriction), the weakness of the ankle in reaching the normal dorsal flexion angle during the swing phase was compensated for by a higher knee and hip flexion, showing a priority in compensating for foot drop. The knee also compensated to protect itself from hyperextension in the terminal stance phase. The hip compensated for itself during the initial swing phase by flexing more to create the necessary moment, normally generated in the terminal stance phase, to advance the leg forward. The hip also compensated for the hyperflexion of the knee in the loading response phase by extending more. In this muscle restriction condition, the weakness of the joint was always compensated for within the kinematic chain, either by the joint itself or by others. 


\subsubsection{CT-Restriction}

For the following discussion, it is important to consider that the CT-restriction restricts the RF, HAM, and VAS muscles in addition to muscles restricted by the C-restriction.

\section{Effect on the ankle motion:}

We observed an increased ankle dorsal flexion during the swing phase (see Figure 3b), which suggests an increased activity of the TA muscle, that is a compensation for the restricted HAM muscles in Van der Krogt's simulations. This action might serve to safely clear the ground, as the knee and hip may not be able to compensate with a higher flexion as they did in C-restriction. The higher ankle dorsal flexion observed in the terminal stance suggests a weak GAS muscle, as also seen in the C-restriction, but in this case, the flexion is higher. The weak GAS is one of the reported effects from restricting the RF muscle in Van der Krogt's simulations.

Effect on the knee motion:

We observed a higher knee extension in the terminal swing phase (see Figure 3d). This might be caused by the decreased activity in the HAM muscles and/or increased activity in the VAS muscles, as reported by Van der Krogt during the swing phase when the VAS muscles are weakened. During the swing phase in the normal gait, the HAM muscles modulate the rate of knee extension, and the VAS muscles act to ensure a complete extension, so as to prepare for the abrupt weight transfer at HC. Weak HAM muscles would fail to regulate the knee extension, and a higher activated VAS would produce a higher knee extension, and compensate for the lower hip flexion that we observed in the majority of subjects at terminal swing (section below). This would otherwise significantly reduce the step length.

\section{Effect on the hip motion:}

We observed a lower hip flexion at pre-swing (see Figure 3f), suggesting weakness in the RF and gracilis (GRA) muscles. These contribute to flexing the hip, to prepare for the swing phase in the normal gait. This partially agrees with Van der Krogt's results, as in that study, a higher activity in the RF muscle during pre-swing and a lower activity in the GRA muscles along the gait cycle were reported as effects of the RF muscle restriction. The observed reduced hip flexion during the initial and middle swing phases also suggests lower activity in the GRA muscle, which normally supports the flexion at this time. This would mean that the hip cannot compensate to clear the ground, as it did in C-restriction. However, as mentioned in the compensations for the ankle, the TA muscle compensates to clear the ground. The observed higher extended hip in the terminal swing phase is the result of the previously mentioned lack of the flexion action of the GRA muscle. As a result of the weak hip flexion during the swing phase, the step length would be shorter than normal.

By comparing the compensation actions manifested in the C- and CT-restrictions, we see a more dynamic compensation activity in C-restriction; this is because the ankle, knee, and hip joints (kinematic chain) are able to compensate for each other, and in some cases even themselves. One of the reasons for this is the severity of the restriction. As the knee and hip joints are minimally affected in C-restriction, they can supply what the overall kinematic chain needs. However, in CT-restriction, the muscle restriction affects the complete kinematic chain; therefore, the body has to prioritize what to compensate. We found that the prioritization includes the prevention of foot drop, knee hyperextension in the terminal stance phase, and knee hyperflexion in the loading response phase, as well as the preservation of the step length. This suggests that the priority is to adapt the gait patterns for safe walking, as also seen in the elderly [24]. Moreover, the evaluation of the significance of every joint motion characteristic in every subject showed that the significance increased at a faster walking speed of $4 \mathrm{~km} / \mathrm{h}$ in both C- and CT-restriction.

Summary of CT-restriction: When the muscles at the calf and thigh area were restricted (CT-restriction), the ankle compensated for itself during the swing phase with a higher dorsal flexion, thereby preventing foot drop. The knee and hip were not able to aid in this task as they did in C-restriction. The hip did not reach the normal flexion range dur- 
ing the swing phase, which reduced the step length. However, some subjects compensated for the higher extended knee in the terminal swing phase to keep the step length. In this muscle restriction condition, the active compensation chain of the C-restriction was not seen. However, the subjects' gait motion appeared to always prioritize safe ambulation, as also seen in the elderly.

\subsection{Joint Motion Compensation and Weakness: Similarities and Differences with the Elderly}

The resultant joint motion of the young adults with the MARTT in C-restriction agreed with several compensation motions and weaknesses seen in the healthy elderly. For instance, the young adults presented a lower ankle plantar flexion in the terminal stance at the time of push-off, as seen in the elderly [11,25]. In addition, a lower dorsal flexion of the ankle during the swing phase was seen in the young adults, which is also commonly known in the elderly as foot drop [26].

Öberg et al. [27] reported the following joint motion changes with aging: increment of knee flexion in the middle stance phase, reduction of knee flexion in the swing phase, and an increment in the hip flexion in the mid-swing phase. In agreement, the young adults with C-restriction showed these motion changes, except for the lower knee flexion during the swing phase. However, the higher knee flexion during the swing phase (as seen in these young adults) has also been found in other studies of the elderly [26,28] as a safety measure to compensate for foot drop.

\subsection{Limitations}

The subject sample that participated in this study is relatively small, and it is possible that there exist other compensation patterns not seen in our analysis. Thus, it is necessary to observe the compensation motions in a much larger subject sample to investigate the universal trend of compensation strategies. Furthermore, a muscle activity analysis is missing to validate what has been discussed in this study regarding which muscles are active or weak, at the moment the observed compensation strategies occur.

To investigate the relationship between the restriction of certain muscle groups and compensation motions, we used MARTT to cause the muscle restriction artificially. However, the congruence between the compensation motions that result from this artificial restriction and the ones that result from natural muscle weakness have to be further analyzed and validated.

\section{Conclusions}

The compensation strategies in human gait appearing under restrictions of the lowerlimb muscles were observed and analyzed in this study. The muscle restriction was achieved using the MARTT, which includes wearable belts for restricting lower-limb muscles. The gait motion was investigated under various conditions of walking speed and muscle restrictions.

The compensation behavior did not appear to be dependent on the walking speed, but the significance of the compensation as evaluated among the subjects increased with the speed. The joint motion weaknesses and compensations seen in the C-restriction agreed with those seen in the elderly, suggesting that human compensation behaviors against muscle weakness have similar purposes, regardless of age. The results of this study provide an essential inspection for understanding the mechanisms of gait compensation against limitations of gait ability.

Supplementary Materials: Supplementary materials can be found at https:/ /www.mdpi.com/ article/10.3390/app11188344/s1.

Author Contributions: Conceptualization, Y.Y., Y.A., S.O. and J.B.U.; methodology, Y.A., S.O. and J.B.U.; software, Y.A. and J.B.U.; validation, Y.A., S.O. and Y.Y.; formal analysis, J.B.U., Y.A. and S.O.; investigation, J.B.U., Y.A. and S.O.; resources, Y.Y.; data curation, J.B.U.; writing-original draft preparation, J.B.U., Y.A.; writing—review and editing, Y.A., J.B.U.; visualization, J.B.U.; supervision, 
Y.A., S.O., Y.Y.; project administration, Y.Y.; funding acquisition, Y.Y. All authors have read and agreed to the published version of the manuscript.

Funding: This research was funded by JSPS KAKENHI grant number 26750121.

Institutional Review Board Statement: The study was conducted according to the guidelines of the Declaration of Helsinki, and approved by the Institutional Review Board of Nagoya University, Japan (approval number 14-4 and date of approval).

Informed Consent Statement: Written informed consent was obtained from all subjects involved in the study.

Data Availability Statement: The subjects' joint angles in every gait section analyzed in this study are compiled and available in the submitted supplementary material; as well as, the statistical analysis of the relevance of the found compensatory motions.

Conflicts of Interest: The authors declare no conflict of interest. The funders had no role in the design of the study; in the collection, analysis, or interpretation of data; in the writing of the manuscript, or in the decision to publish the results.

Abbreviations
$\begin{array}{ll}\text { The following abbreviations are used in this manuscript: } \\ \text { RF } & \text { rectus femoris } \\ \text { VAS } & \text { vastii } \\ \text { BFS } & \text { biceps femoris short head } \\ \text { GAS } & \text { gastrocnemius } \\ \text { SO } & \text { soleus } \\ \text { TA } & \text { tibialis anterior } \\ \text { HAM } & \text { hamstring } \\ \text { ILPS } & \text { iliopsoas } \\ \text { MARTT } & \text { Muscle Activity Restriction Taping Technique } \\ \text { MTC } & \text { minimum toe clearance } \\ \text { HC } & \text { heel contact } \\ \text { TO } & \text { toe off } \\ \text { C-restriction } & \text { calf restriction } \\ \text { CT-restriction } & \text { calf and thigh restriction }\end{array}$

\section{References}

1. Hollmann, W.; Strüder, H.; Tagarakis, C.; King, G. Physical activity and the elderly. Eur. J. Cardiovasc. Prev. Rehabil. 2007, 14, 730-739. [CrossRef]

2. Lamoureux, E.L.; Sparrow, W.A.; Murphy, A.; Newton, R.U. Differences in the neuromuscular capacity and lean muscle tissue in old and older community-dwelling adults. J. Gerontol. A Biol. Sci. Med. Sci. 2001, 56, M381-M385. [CrossRef] [PubMed]

3. Frontera, W.R.; Hughes, V.A.; Fielding, R.A.; Fiatarone, M.A.; Evans, W.J.; Roubenoff, R. Aging of skeletal muscle: A 12-year longitudinal study. J. Appl. Physiol. 2000, 88, 1321-1326. [CrossRef] [PubMed]

4. Hayashida, I.; Tanimoto, Y.; Takahashi, Y.; Kusabiraki, T.; Tamaki, J. Correlation between muscle strength and muscle mass, and their association with walking speed, in community-dwelling elderly Japanese individuals. PLoS ONE 2014, 9, e111810.

5. McCormick, R.; Vasilaki, A. Age-related changes in skeletal muscle: Changes to life-style as a therapy. Biogerontology 2018, 19, 519-536. [CrossRef] [PubMed]

6. Akima, H.; Kano, Y.; Enomoto, Y.; Ishizu, M.; Okada, M.; Oishi, Y.; Katsuta, S.; Kuno, S. Muscle function in 164 men and women aged 20-84 year. Med. Sci. Sports Exerc. 2001, 33, 220-226. [CrossRef] [PubMed]

7. Janssen, I.; Heymsfield, S.B.; Wang, Z.M.; Ross, R. Skeletal muscle mass and distribution in 468 men and women aged 18-88 year. J. Appl. Physiol. 2000, 89, 81-88. [CrossRef] [PubMed]

8. Ikezoe, T.; Mori, N.; Nakamura, M.; Ichihashi, N. Age-related muscle atrophy in the lower extremities and daily physical activity in elderly women. Arch. Gerontol. Geriatr. 2011, 53, 153-157. [CrossRef]

9. Kirkwood, R.N.; Trede, R.G.; Moreira, B.; Kirkwood, S.A.; Pereira, L.S. Decreased gastrocnemius temporal muscle activation during gait in elderly women with history of recurrent falls. Gait Posture 2011, 34, 60-64. [CrossRef]

10. Schmitz, A.; Silder, A.; Heiderscheit, B.; Mahoney, J.; Thelen, D. Differences in lower-extremity muscular activation during walking between healthy older and young adults. J. Electromyogr. Kinesiol. 2009, 19, 1085-1091. [CrossRef]

11. Begg, R.K.; Sparrow, W.A. Ageing effects on knee and ankle joint angles at key events and phases of the gait cycle. J. Med. Eng. Technol. 2006, 30, 382-389. [CrossRef] [PubMed] 
12. Aniansson, A.; Grimby, G.; Hedberg, M. Compensatory muscle fiber hypertrophy in elderly men. J. Appl. Physiol. 1992, 73, 812-816. [CrossRef] [PubMed]

13. Stewart, J.D. Foot drop: Where, why and what to do? Pract. Neurol. 2008, 8, 158-169. [CrossRef] [PubMed]

14. Pirker, W.; Katzenschlager, R. Gait disorders in adults and the elderly: A clinical guide. Wien Klin. Wochenschr. 2017, 129, 81-95. [CrossRef] [PubMed]

15. DeVita, P.; Hortobagyi, T. Age causes a redistribution of joint torques and powers during gait. J. Appl. Physiol. 2000, 88, 1804-1811. [CrossRef]

16. Mill, P.; Barret, R.S. Swing phase mechanics of healthy young and elderly men. Hum. Mov. Sci. 2001, 20, 427-446. [CrossRef]

17. Van der Krogt, M.M.; Delp, S.L.; Schwartz, M.H. How robust is human gait to muscle weakness? Gait Posture 2012, 36, 113-119. [CrossRef]

18. Ullauri, J.B.; Akiyama, Y.; Okamoto, S.; Yamada, Y. Technique to reduce the minimum toe clearance of young adults during walking to simulate the risk of tripping of the elderly. PLOS ONE 2019, 14, e0217336. [CrossRef]

19. Abe, T.; Kearns, C.; Sato, Y. Muscle size and strength are increased following walk training with restricted venous blood flow from the leg muscle, Kaatsu-walk training. J. Appl. Physiol. 2006, 100, 1460-1466. [CrossRef] [PubMed]

20. Abe, T.; Sakamaki, M.; Fujita, S.; Ozaki, H.; Sugaya, M.; Sato, Y. Effects of low-intensity walk training with restricted leg blood flow on muscle strength and aerobic capacity in older adults. J. Geriatr. Phys. Ther. 2010, 33, 34-40.

21. Karst, G.M.; Hageman, P.A.; Jones, T.F.; Bunner, S.H. Reliability of foot trajectory measures within and between testing sessions J. Gerontol. 1999, 54, 343-347. [CrossRef] [PubMed]

22. Hamacher, D.; Hamacher, D.; Schega, L. Towards the importance of minimum toe clearance in level ground walking in a healthy elderly population. Gait Posture 2014, 40, 727-729. [CrossRef]

23. Perry, J.; Burnfield, J.M. Gait Analysis: Normal and Pathological Function, 2nd ed.; Slack Inc.: Thorofare, NJ, USA, $2010 ;$ pp. 53-119.

24. Winter, D.A.; Patla, A.E.; Frank, J.S.; Walt, S.E. Biomechanical walking pattern changes in the fit and healthy elderly. Phys. Ther. 1990, 70, 340-347. [CrossRef] [PubMed]

25. Kerrigan, D.C.; Todd, M.K.; Della Croce, U.; Lipsitz, L.A.; Collins, J.J. Biomechanical gait alterations independent of speed in the healthy elderly: Evidence for specific limiting impairments. Arch. Phys. Med. Rehabil. 1998, 79, 317-322. [CrossRef]

26. Mills, P.; Barrett, R.; Morrison, S. Toe clearance variability during walking in young and elderly men. Gait Posture 2008, 28, 101-107. [CrossRef]

27. Oberg, T.; Karsznia, A.; Oberg, K. Joint angle parameters in gait: Reference data for natural subjects, 10-79 of age. J. Rehabil. Res. Dev. 1994, 31, 199-213.

28. Prince, F.; Corriveau, H.; Hebert, R.; Winter, D.A. Gait in the elderly. Gait Posture 1997, 5, 128-135. [CrossRef] 